HUPD-0103

\title{
Dynamical Fermion Masses Under the Influence of Kaluza-Klein Fermions in Randall-Sundrum Background *
}

\author{
Hiroyuki Abe ${ }^{1}$, Tomohiro Inagaki ${ }^{2}$, and Taizo Muta ${ }^{3}$ \\ ${ }^{1}$ Department of Physics, Hiroshima University, \\ Higashi-Hiroshima, Hiroshima 739-8526, Japan \\ E-mail: abe@theo.phys.sci.hiroshima-u.ac.jp \\ ${ }^{2}$ Research Institute for Information Science and Education, Hiroshima University, \\ Higashi-Hiroshima, Hiroshima 739-8521, Japan \\ E-mail: inagaki@hiroshima-u.ac.jp \\ 3 Department of Physics, Hiroshima University, \\ Higashi-Hiroshima, Hiroshima 739-8526, Japan \\ E-mail: muta@hiroshima-u.ac.jp
}

\begin{abstract}
The dynamical fermion mass generation on the D3-brane in the RandallSundrum space-time is discussed in a model with bulk fermions in interaction with fermions on the branes. It is found that the dynamical fermion masses are generated at the natural (R.-S.) radius of the compactified extra space and may be made small compared with masses of the Kaluza-Klein modes which is of order of $\mathrm{TeV}$.
\end{abstract}

04.50.th,04.60.-m,11.15.Pg,11.30.Qc

Typeset using REVTEX

*This paper is dedicated to the 60th birthday of Professor Hagen Kleinert who is the good old friend of one of the authors. 


\section{INTRODUCTION}

It is an interesting idea to assume an existence of the extra-dimensional space which eventually compactifies leaving our 4-dimensional space-time as a real world. [1] The recent proposal [2,3] for the mass scale of the compactified space to be much smaller than the Planck scale gave a strong impact on the onset of studying phenomenological evidences of extra-dimensional effects. [1] There is a crucial problem, however, how such large extra dimensions are stabilized. Recently, L. Randall and R. Sundrum [5] (R.-S.) gave an alternative to the large extra dimension scenario. By introducing a specific curved bulk space they succeeded to have a mass scale much smaller than the Planck scale without relying on the fine-tuning.

In our present analysis we introduce bulk fermions in the R.-S. space-time [6] and see what effects could be observed. The bulk fermions interact with themselves as well as with fermions on the 4-dimensional branes through the exchange of the graviton and its KaluzaKlein excited modes, or through the exchange of gauge bosons which may be assumed to exist in the bulk. [6] The interactions among fermions generated as a result of the exchange of all the Kaluza-Klein excited modes of the graviton or gauge bosons may be expressed as effective four-fermion interactions. [4, 7,8] According to the four-fermion interactions we expect that the dynamical generation of fermion masses will take place. In the present communication we look for a possibility of the dynamical fermion mass generation under the influence of the bulk fermions through the effective four-fermion interactions in the R.S. background. In the R.-S. background fermion mass terms are forbidden by the $S^{1} / \mathbf{Z}_{2}$ symmetry. The possible source of fermion masses on the branes is two-fold, i. e. the dynamically generated fermion masses and masses of the Kaluza-Klein excited modes of the bulk fermions. The mass of the Kaluza-Klein excited modes is known to be of order of TeV. 6]

In section [I] we will review the dynamical fermion mass generation with bulk fermions in torus compactified case [9] because most of the technical parts can be summarized in the torus (flat) extra dimension case. And in section [II we proceed to the case of the R.-S. spacetime, the main part of this paper. In the section it is found that the dynamical fermion masses are generated at the natural (R.-S.) radius of the compactified extra dimensional space and may be made small compared with masses of the Kaluza-Klein modes which is of order of $\mathrm{TeV}$ because of the presence of the Randall-Sundrum warp factor.

\section{FLAT EXTRA SPACE}

We assume an existence of 5-dimensional bulk fermions $\psi$ in interaction with fermions $L$ on the 4-dimentional brane. Effective interactions among these fermions can be given in the form of the four-fermion interaction. In fact it is known that the exchange of the KaluzaKlein excited modes of the bulk graviton results in effective four-fermion interactions. [4] After the Fierz transformation on the four-fermion interactions we generate the transitiontype interactions. Accordingly we start with the following Lagrangian for our model 9]

$$
\mathcal{L}^{(5)}=\bar{\psi} i \gamma^{M} \partial_{M} \psi+\left[\bar{L} i \gamma^{\mu} \partial_{\mu} L+g^{2} \bar{\psi} \gamma^{M} L \bar{L} \gamma_{M} \psi\right] \delta\left(x^{4}\right)
$$


where $g$ is the coupling constant with mass dimension $-3 / 2$ and index $M$ runs from 0 to 4 while index $\mu$ runs from 0 to 3 . Fermions $\psi$ and $L$ are assumed to be of $N_{f}$ components.

After neglecting tensor interactions and introducing auxiliary field $\sigma \sim \bar{\psi} L$, we compactify the bulk space on torus with radius $R$. Our 4-dimensional Lagrangian is rewritten as

$$
\mathcal{L}^{(4)}=\bar{\Psi}(M+i \not \partial) \Psi-|\sigma|^{2}
$$

where $\Psi^{t} \equiv\left(L, \psi_{0}, \psi_{1}, \psi_{-1}, \psi_{2}, \psi_{-2} \cdots\right)$ and

$$
M \equiv\left(\begin{array}{ccccccc}
0 & m^{*} & m^{*} & m^{*} & m^{*} & m^{*} & \cdots \\
m & 0 & 0 & 0 & 0 & 0 & \cdots \\
m & 0 & \frac{1}{R} & 0 & 0 & 0 & \cdots \\
m & 0 & 0 & -\frac{1}{R} & 0 & 0 & \cdots \\
m & 0 & 0 & 0 & \frac{2}{R} & 0 & \cdots \\
m & 0 & 0 & 0 & 0 & -\frac{2}{R} & \cdots \\
\vdots & \vdots & \vdots & \vdots & \vdots & \vdots & \ddots
\end{array}\right), \quad(m=g \sigma / \sqrt{2 \pi R})
$$

If $\sigma$ acquires a non-vanishing vcuum expectation value, we replace $\sigma$ in $m$ by its vacuum expectation value $\langle\sigma\rangle$, i. e. $m=N g\langle\sigma\rangle$. The eigenvalues of matrix $M$ determine the masses of 4-dimensional fermions. Obviously we find that the lightest eigenvalue is given by

$$
\lambda_{ \pm 0}= \pm|m| \quad \text { for } \quad|m| \ll 1 / R
$$

Thus we conclude that within our scheme there is a possibility of having the light fermion masses which is much smaller than the mass of the Kaluza-Klein modes of the bulk fermion. By performing the path-integration for fermion field $\Psi$ we find the effective potential for $\sigma$ in the leading order of the $1 / N_{f}$ expansion:

$$
\begin{aligned}
V(\sigma)=|\sigma|^{2} & -\frac{1}{2 \pi^{2}} \int_{0}^{\Lambda} d x x^{3} \ln \left[x^{2}+|m|^{2}(\pi x R) \operatorname{coth}(\pi x R)\right] \\
& -\frac{1}{2 \pi^{2}} \sum_{j=1}^{\infty} \int_{0}^{\Lambda} d x x^{3} \ln \left[x^{2}+\left(\frac{j}{R}\right)^{2}\right]
\end{aligned}
$$

The gap equation to determine the vacuum expectation value $\langle|\sigma|\rangle$ of $|\sigma|$ reads

$$
\frac{\partial V(\sigma)}{\partial|\sigma|}=2|\sigma|\left\{1-\frac{g^{2}}{2 \pi^{2}} \int_{0}^{\Lambda} d x \frac{x^{3}}{2 x \tanh (\pi x R)+g^{2}|\sigma|^{2}}\right\}=0 .
$$

By numerical observation of Eq. (2.6) we find that there exists a non-trivial solution for $|\sigma|$ for a suitable range of parameters $g$ and $R$ and the solution corresponds to the true minimum of the effective potential. Accordingly the fermion mass is generated dynamically. Here the auxiliary field $\sigma$ (or the composite field $\bar{L} \psi$ ) acquires a vacuum expectation value. Moreover it is easily confirmed that the phase transition associated with this symmetry breaking is of second order.

As was shown in Eq. (2.4) the lowest fermion mass on the 4-dimensional brane is $m=$ $N g\langle|\sigma|\rangle$ where $\langle|\sigma|\rangle$ is determined by solving Eq. (2.6). The critical curve which represents 
the critical radius as a function of the coupling constant is shown in Fig. 1 as the curve for $m=0$. If we assume $1 / R \sim \mathrm{TeV}$, light $(<\mathrm{TeV})$ fermions are obtained in the region between the solid line and the dot-dashed line in the Fig. 1 because it is natural that $R \Lambda \sim 1$. We, however, have no idea to justify $1 / R \sim \mathrm{TeV}$ and so we need to introduce a certain mechanism such as the Randall-Sundrum model in the next section.

\section{WARPED EXTRA SPACE}

In theories with large $\left(\gg M_{p l}^{-1}\right)$ extra dimemsions people wonder how such large radii are stabilized. We have not yet come across with any satisfactory answer, while important results in many models depend essentially on the largeness of the radius as in the previous section. L. Randall and R. Sundrum [5] noticed that the existence of the brane leads to the curved bulk space and proposed the so-called Randall-Sundrum (R.-S.) model. As is shown in the Fig. 2 their model has two D3-branes on the $S_{1} / \mathbf{Z}_{\mathbf{2}}$ orbifold fixed points and the $A d S_{5}$ between these branes:

$$
d s^{2} \equiv G_{M N} d x^{M} d x^{N}=e^{-2 k b_{0}|y|} \eta_{\mu \nu} d x^{\mu} d x^{\nu}+b_{0}^{2} d y^{2}
$$

where $k \sim M_{p l}$ is the gravity scale, $b_{0}^{-1} \sim k / 24 \pi$ is the compactification scale. One of the most important results is the $e^{-\frac{1}{2} k b_{0}}$ (warp factor) suppression of the K.-K. masses of the bulk fields. [6]

\section{A. Bulk Fermions in R.-S. Background}

Following Chang et al. [6] we derive the mode expansion of the bulk fermion in the R.-S. space-time such that

$$
\begin{gathered}
\psi(x, y)=\frac{e^{\frac{3}{2} k b_{0}|y|}}{\sqrt{b_{0}}} \sum_{n}\left[\psi_{L}^{(n)}(x) \xi(y)+\psi_{R}^{(n)}(x) \eta(y)\right], \\
\left\{\begin{array}{l}
\xi(y)=\sqrt{\frac{k b_{0}}{1-e^{-\frac{1}{2} k b_{0}}}} e^{-\frac{1}{2} k b_{0}\left|\frac{1}{2}-y\right|} \sin \frac{m_{n}}{k b_{0}}\left(1-e^{k b_{0}|y|}\right) \\
\eta(y)=\sqrt{\frac{k b_{0}}{1-e^{-\frac{1}{2} k b_{0}}}} e^{-\frac{1}{2} k b_{0}\left|\frac{1}{2}-y\right|} \cos \frac{m_{n}}{k b_{0}}\left(1-e^{k b_{0}|y|}\right)
\end{array}\right.
\end{gathered}
$$

where $m_{n} \equiv n \pi k b_{0} /\left(e^{\frac{1}{2} k b_{0}}-1\right)$ is $b_{0}$ times the mass of the $\mathrm{n}$-th K.-K. mode. With this expansion the kinetic terms of the K.-K. modes become

$$
\mathcal{L}_{\text {bluk }}^{(4)}=\int d y E \bar{\psi} i \gamma^{M} D_{M} \psi=\sum_{n}\left[\bar{\psi}^{(n)} i \not \partial \psi^{(n)}-\frac{m_{n}}{b_{0}} \bar{\psi}^{(n)} \psi^{(n)}\right]
$$

In the following we apply previous results of our model with bulk fermions in the R.-S. background. 


\section{B. Four-Fermion Interaction Model in R.-S. Background}

Now we apply the previous torus-compactified model (2.1) to the case in the R.-S. background:

$$
E_{\bar{M}}^{M}=\left(\begin{array}{cc}
e^{k b_{0}|y|} \eta_{\bar{\mu}}^{\mu} & 0 \\
0 & b_{0}^{-1}
\end{array}\right)
$$

where $E_{\bar{M}}^{M}$ is the vielvein whose square becomes the metric $G_{M N}$. We introduce the bulk fermion $\psi$ which propagates in the whole five dimensional space as in the torus case, while two brane fermions $L_{i}(i=1,2)$ propagate on an i-th brane shown in the Fig. 2 respectively.

We start with the Lagrangian in 5-dimensions,

$$
\begin{gathered}
\mathcal{L}^{(5)}=E \bar{\psi} i \gamma^{M} D_{M} \psi+E_{(1)}\left[\bar{L}_{1} i \gamma^{\mu} \partial_{\mu} L_{1}+\frac{g_{1}^{2}}{N_{f}} \bar{\psi} \gamma^{M} L_{1} \bar{L}_{1} \gamma_{M} \psi\right] \delta\left(x^{4}\right) \\
+E_{(2)}\left[\overline{L_{2}} i \gamma^{\mu} \partial_{\mu} L_{2}+\frac{g_{2}^{2}}{N_{f}} \bar{\psi} \gamma^{M} L_{2} \overline{L_{2}} \gamma_{M} \psi\right] \delta\left(x^{4} \frac{1}{2}\right)
\end{gathered}
$$

where $x^{M}=\left(x^{\mu}, x^{4}=y\right), \mu=(0,1,2,3), g_{i}$ 's are the four-fermion couplings $\left(\left[g_{i}\right]=-3 / 2\right)$ and $N_{f}$ is the number of the component of $\psi$ and $L_{i}$ 's. Note that the bulk mass term is removed by the $S^{1} / \mathbf{Z}_{\mathbf{2}}$ projection. Because Lorentz covariance should be preserved, we neglect the tensor (vector) interactions and obtain

$$
\begin{aligned}
\mathcal{L}^{(5)}=E \bar{\psi} i \gamma^{M} D_{M} \psi+\left[\bar{L}_{1} i \gamma^{\bar{\mu}} \partial_{\bar{\mu}} L_{1}+\frac{g_{1}^{2}}{N_{f}} \bar{\psi} \gamma_{5} L_{1} \bar{L}_{1} \gamma_{5} \psi\right] \delta\left(x^{4}\right) \\
+e^{-2 k b_{0}}\left[e^{\frac{1}{2} k b_{0}} \overline{L_{2}} i \gamma^{\bar{\mu}} \partial_{\bar{\mu}} L_{2}+\frac{g_{2}^{2}}{N_{f}} \bar{\psi} \gamma_{5} L_{2} \bar{L}_{2} \gamma_{5} \psi\right] \delta\left(x^{4} \frac{1}{2}\right)
\end{aligned}
$$

After chiral rotation $\psi \rightarrow e^{i \frac{\pi}{4} \gamma^{5}} \psi$ and $L_{i} \rightarrow e^{i \frac{\pi}{4} \gamma^{5}} L_{i}$, we introduce auxiliary fields $\sigma_{i}$ 's to obtain

$$
\begin{aligned}
\mathcal{L}^{(5)}= & E \bar{\psi} i \gamma^{M} \partial_{M} \psi+\left[\overline{L_{1}} i \not \partial L_{1}-N_{f}\left|\sigma_{1}\right|^{2}+\left(g_{1} \sigma_{1} \bar{\psi} L_{1}+\text { h.c. }\right)\right] \delta\left(x^{4}\right) \\
& +e^{-2 k b_{0}}\left[e^{\frac{1}{2} k b_{0}} \overline{L_{2}} i \not \partial L_{2}-N_{f}\left|\sigma_{2}\right|^{2}+\left(g_{2} \sigma_{2} \bar{\psi} L_{2}+\text { h.c. }\right)\right] \delta\left(x^{\underline{4}} \frac{1}{2}\right) .
\end{aligned}
$$

Now we substitute the mode expansion of $\psi$ of Eq. (3.2) into the Lagrangian and integrate it over the extra-dimension. Taking $e^{-\frac{3}{4} k b_{0}} L_{2} \rightarrow L_{2}$ and $e^{-k b_{0}} \sigma_{2} \rightarrow \sigma_{2}$, Lagrangian in 4dimension becomes

$$
\begin{aligned}
\mathcal{L}^{(4)} \equiv & \int d y \mathcal{L}^{(5)} \\
= & \sum_{n}\left[\bar{\psi}^{(n)} i \not \partial \psi^{(n)}-\frac{n}{R} \bar{\psi}^{(n)} \psi^{(n)}\right]+\bar{L}_{1} i \not \partial L_{1}+\bar{L}_{2} i \not \partial L_{2}-N_{f}\left(\left|\sigma_{1}\right|^{2}+\left|\sigma_{2}\right|^{2}\right) \\
& +\sum_{n}\left(\mu_{1} \bar{\psi}_{R}^{(n)} L_{1}+\text { h.c. }\right)+\sum_{n}\left((-1)^{n} \mu_{2} \bar{\psi}_{R}^{(n)} L_{2}+\text { h.c. }\right),
\end{aligned}
$$

where 


$$
\mu_{1} \equiv \frac{g_{1} \sigma_{1}}{\sqrt{b_{0}}}, \quad \mu_{2} \equiv \frac{g_{2} \sigma_{2}}{\sqrt{b_{0}}}, \quad R \equiv \frac{b_{0}}{m_{1}}=\frac{e^{\frac{1}{2} k b_{0}}-1}{\pi k} \sim(\mathrm{TeV})^{-1}
$$

Here we note that the mass of the 1 st K.-K. excited mode $1 / R$ is of order of $\mathrm{TeV}$ without fine-tuning because of the warp factor in the R.-S. metric, and it is the only dependence on the factor.

After integrating out all fermionic degrees of freedom, we obtain the effective potential for $\sigma_{i}\left(\mu_{i}\right)$ as follows:

$$
\begin{aligned}
& \bar{V}\left(\mu_{1} / \Lambda, \mu_{2} / \Lambda\right) \\
& =\left[V\left(\mu_{1} / \Lambda, \mu_{2} / \Lambda\right)-V(0,0)\right] / \Lambda^{4} \\
& =2 \pi R \Lambda\left[\frac{\left(\mu_{1} / \Lambda\right)^{2}}{g_{1}^{2} \Lambda^{3}}+\frac{\left(\mu_{2} / \Lambda\right)^{2}}{g_{2}^{2} \Lambda^{3}}\right] \\
& \quad-\frac{1}{2 \pi^{2}} \int_{0}^{1} d z z^{3} \ln \left[1+\frac{\pi R \Lambda}{z}\left\{\left(\frac{\mu_{1}}{\Lambda}\right)^{2}+\left(\frac{\mu_{2}}{\Lambda}\right)^{2}\right\} \operatorname{coth}(\pi z R \Lambda)+\frac{\pi^{2}}{2}(R \Lambda)^{2}\left(\frac{\mu_{1}}{\Lambda}\right)^{2}\left(\frac{\mu_{2}}{\Lambda}\right)^{2}\right] .
\end{aligned}
$$

\section{Behavior of the Vacuum $\left\langle\mu_{i}\right\rangle$}

The behavior of the vacuum is determined by solving the gap equations $(i, j=1,2 ; i \neq j)$ :

$$
\begin{aligned}
& \frac{\partial \bar{V}}{\partial\left(\mu_{i} / \Lambda\right)} \\
& =R \Lambda \frac{\mu_{i}}{\Lambda}\left\{\frac{4 \pi}{g_{i}^{2} \Lambda^{3}}-\frac{1}{2 \pi} \int_{0}^{1} d z z^{3} \frac{\pi R \Lambda \mu_{j}^{2} \tanh (\pi z R \Lambda)+2}{z\left\{1+\frac{\pi^{2}}{2}(R \Lambda)^{2}\left(\frac{\mu_{1}}{\Lambda}\right)\left(\frac{\mu_{2}}{\Lambda}\right)\right\} \tanh (\pi z R \Lambda)+\pi R \Lambda\left\{\left(\frac{\mu_{1}}{\Lambda}\right)+\left(\frac{\mu_{2}}{\Lambda}\right)\right\}}\right\} \\
& =0 .
\end{aligned}
$$

The system has a second order phase transition and $\left\langle\mu_{2}\right\rangle$ as a function of $g_{1}$ and $g_{2}$ is shown in the Fig. 3 for $R \Lambda=1$. If $g_{i} \gtrsim g_{i \text {,critical }}$, we see $\left\langle\mu_{i}\right\rangle \gtrsim 0$. The phase strucutre of this system is summerized in the Fig. 6 .

\section{Effective Theory on $y=\frac{1}{2}$ brane}

Now we realize that $\mu_{1}$ and $\mu_{2}$ can have non-zero vacuum expectation value and Lagrangian (3.9) has mixing term $\bar{\psi}_{R} L_{i}+$ h.c.. The physics on the $i=2$ brane is examined by integrating out the invisible field $L_{1}$. Setting $\sqrt{2} \psi_{L}^{(n)} \equiv N^{(n)}-M^{(n)}$ and $\sqrt{2} \psi_{R}^{(n)} \equiv N^{(n)}+M^{(n)}(n \neq 0)$, the effective Lagrangian on the $y=\frac{1}{2}$ brane is obtained as follows:

$$
\mathcal{L}_{\mathrm{eff}}^{(4)}=\bar{\Theta}_{2}\left[i \not \partial+M_{2}+\left|\mu_{1}\right|^{2} P\right] \Theta_{2}
$$

where $\Theta_{2}^{T} \equiv\left(L_{2}, \psi_{R}^{(0)}, N^{(1)}, M^{(1)}, N^{(2)}, M^{(2)} \ldots\right)$ and 


$$
\begin{gathered}
M_{2} \equiv\left(\begin{array}{ccccccc}
0 & \mu_{2}^{*} & -\mu_{2}^{*} & -\mu_{2}^{*} & \mu_{2}^{*} & \mu_{2}^{*} & \ldots \\
\mu_{2} & 0 & 0 & 0 & 0 & 0 & \ldots \\
-\mu_{2} & 0 & \frac{1}{R} & 0 & 0 & 0 & \ldots \\
-\mu_{2} & 0 & 0 & -\frac{1}{R} & 0 & 0 & \ldots \\
\mu_{2} & 0 & 0 & 0 & \frac{2}{R} & 0 & \ldots \\
\mu_{2} & 0 & 0 & 0 & 0 & -\frac{2}{R} & \ldots \\
\vdots & \vdots & \vdots & \vdots & \vdots & \vdots & \ddots
\end{array}\right), \\
P \equiv\left(\begin{array}{ccccccc}
0 & 0 & 0 & 0 & 0 & 0 & \ldots \\
0 & (i \not \partial)^{-1} & (i \not \partial)^{-1} & (i \not \partial)^{-1} & (i \not \partial)^{-1} & (i \not \partial)^{-1} & \ldots \\
0 & (i \not \partial)^{-1} & (i \not \partial)^{-1} & (i \not \partial)^{-1} & (i \not \partial)^{-1} & (i \not \partial)^{-1} & \ldots \\
0 & (i \not \partial)^{-1} & (i \not \partial)^{-1} & (i \not \partial)^{-1} & (i \not \partial)^{-1} & (i \not \partial)^{-1} & \ldots \\
0 & (i \not \partial)^{-1} & (i \not \partial)^{-1} & (i \not \partial)^{-1} & (i \not \partial)^{-1} & (i \not \partial)^{-1} & \ldots \\
0 & (i \not \partial)^{-1} & (i \not \partial)^{-1} & (i \not \partial)^{-1} & (i \not \partial)^{-1} & (i \not \partial)^{-1} & \ldots \\
\vdots & \vdots & \vdots & \vdots & \vdots & \vdots & \ddots
\end{array}\right) .
\end{gathered}
$$

We have seen in the previous subsection that the dynamical fermion mass generation with $\psi$ is of the second order phase transition, and we find the parameter region of $\left(g_{1}, g_{2}\right)$ for $\left\langle\mu_{2}\right\rangle \lesssim \Lambda=1 / R \sim \mathrm{TeV},\left\langle\mu_{1}\right\rangle=0$ for the cut-off $\Lambda=1 / R$. Thus in Eq. (3.13) we have a posibility to get a light Dirac fermion on the $i=2$ brane.

\section{CONCLUSION}

We have found within our model (3.6) that the dynamically generated fermion mass is much smaller than the Planck scale because of the presence of the mass scale $1 / R \sim \mathrm{TeV}$ generated by the Randall-Sundrum warp factor. Furthermore, in spite of the presence of the mass scale $1 / R \sim \mathrm{TeV}$ in the theory the fermion masses on the 4-dimensional brane can be made smaller than this scale as a consequence of the interaction among the bulk and brane fermions: the mixing of the brane fermions with the bulk fermions does not lead to the lightest fermion masses of order $1 / R$ and the dynamically generated fermion masses are not of order $1 / R$ but of order $\left\langle\mu_{2}\right\rangle$ because of the same mechanism as Eq. (2.4). This result is obtained because the dynamical fermion masses generated under the second-order phase transition are small irrespectively of $1 / R$ near the critical radius. In our model the possibility of having low mass fermions resulted from the dynamical origin. This mechanism is quite different from the ones in other approaches in which low mass fermions are expected to show up as a result of the kinematical origins. 10 14

Our outlook is summarized as in the following four items. The first item is whether the K.-K. gauge boson (or graviton) exchange induce suitable effective four-fermion interactions, the second is what the physics on the visible $(i=2)$ brane with $\left\langle\mu_{1}\right\rangle \neq 0$ is, the third is how we stabilize the radius $b_{0}=24 \pi k^{-1}$ with the pressure of the bulk fermion $\psi$, [15 17] and the last is the extension of our model to electroweak symmetry breaking. [7,8,18, 20] 17

\footnotetext{
${ }^{1}$ In the Ref. [20], the dynamical generation of fermion masses as well as electroweak symmetry breaking in the Randall-Sundrum scenario is studied. This paper also presents a scenario which
} 


\section{REFERENCES}

[1] Th. Kaluza, Sitzungsber. d. Preuss. Akad. d. Wiss. p.966 (1921); O. Klein, Zeitsch. f. Phys. 37, 895 (1926).

[2] I. Antoniadis, Phys. Lett. B 246, 377 (1990); I. Antoniadis and K. Benakli, Phys. Lett. B 326, 69 (1994).

[3] N. Arkani-Hamed, S. Dimopoulos and G. Dvali, Phys. Lett. B429, 263 (1998); Phys. Rev. D59, 086004 (1999); I. Antoniadis, N. Arkani-Hamed, S. Dimopoulos and G. Dvali, Phys. Lett. B 436, 257 (1998).

[4] T. Han, J. D. Lykken and R-J. Zhang, Phys. Rev. D59, 105006 (1999).

[5] L. Randall and R. Sundrum, Phys. Rev. Lett. 83, 3370 (1999).

[6] S. Chang, J. Hisano, H. Nakano, N. Okada and M. Yamaguchi, Phys. Rev. D62, 084025 (2000).

[7] B. A. Dobrescu, Phys. Lett. B461, 99 (1999).

[8] H-C. Cheng, B. A. Dobrescu and C. T. Hill, Nucl. Phys. B 589, 249 (2000); A. B. Kobakhidze, hep-ph/9904203.

[9] H. Abe, H. Miguchi and T. Muta, Mod. Phys. Lett. A15, 445 (2000).

[10] N. Arkani-Hamed, S. Dimopoulos, G. Dvali and J. March-Russel, hep-ph/9811448.

[11] K. R. Dienes, E. Dudas and T. Gherghetta, Nucl. Phys. B557, 25 (1999).

[12] R. N. Mohapatra, S. Nandi and A. Perez-Lorenzana, Phys. Lett. B466, 115 (1999).

[13] A. Das, and C. W. Kong, Phys. Lett. B470, 149 (1999).

[14] K. Yoshioka, Mod. Phys. Lett. A15, 29 (2000).

[15] S. A. Gundersen and F. Ravndal, Ann. Phys. 182, 90 (1988).

[16] H. Abe, J. Hashida, T. Muta and A. Purwanto, Mod. Phys. Lett. A14, 1033 (1999).

[17] W. D. Goldberger and M. B. Wise, Phys. Rev. Lett. 83, 4922 (1999).

[18] N. Arkani-Hamed, H.-C. Cheng, B. A. Dobrescu and L. J. Hall, Phys. Rev. D62, 096006 (2000).

[19] M. Hashimoto, M. Tanabashi and K. Yamawaki, hep-ph/0010260

[20] N. Rius and V. Sanz, hep-ph/0103086.

\section{ACKNOWLEDGMENTS}

The authors would like to thank Hironori Miguchi, Koichi Yoshioka (Kyoto U.), Masahiro Yamaguchi (Tohoku U.) and Hiroaki Nakano (Niigata U.) for fruitful discussions and correspondences. They are also indebted to Tak Morozumi for useful comments. The present work is supported financially by the Monbusho Grant, Grant-in-Aid for Scientific Research (C) with contract number 11640280 .

can explain the observed hierarchy of fermion masses. 


\section{FIGURES}

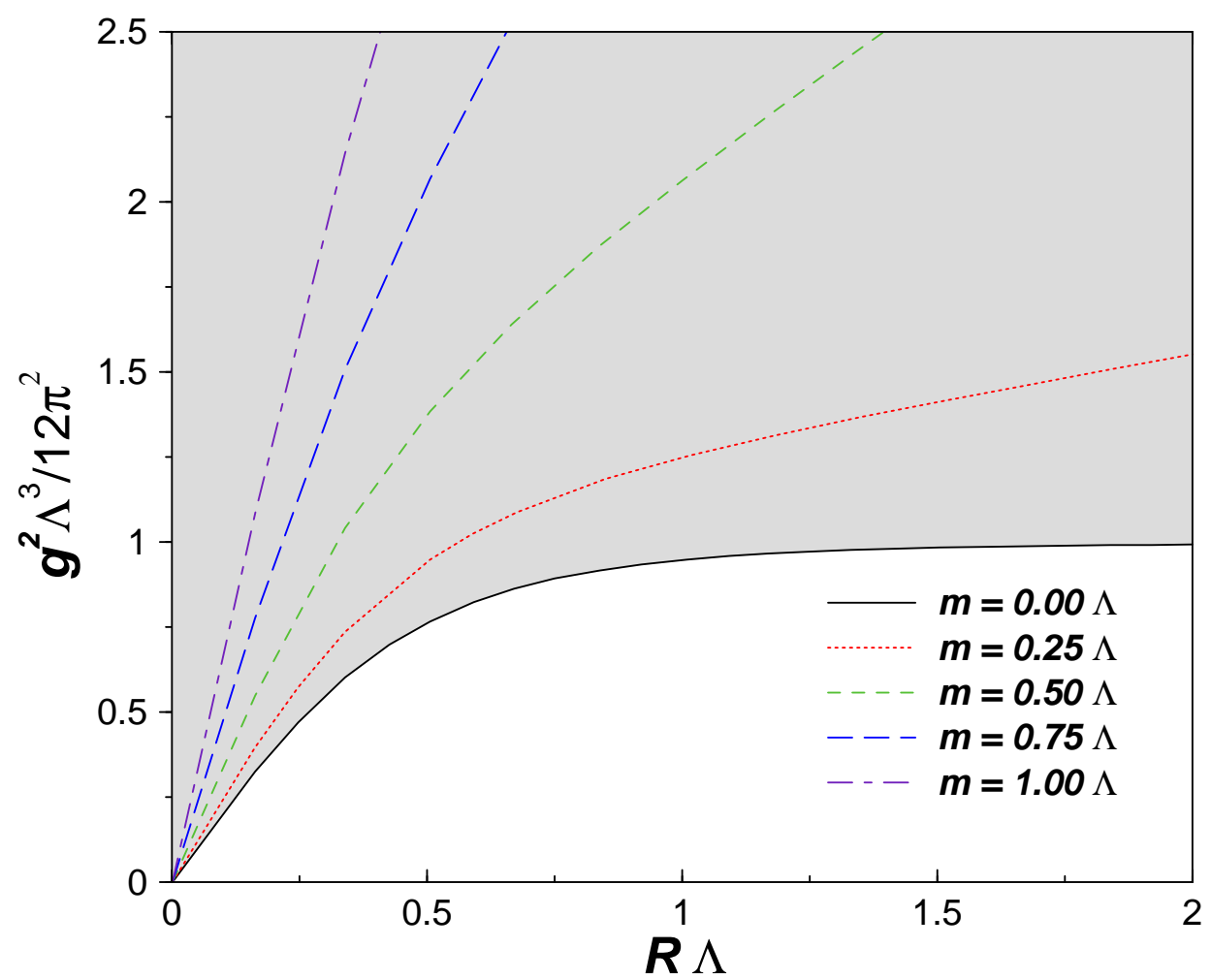

FIG. 1. Critical radius as a function of $g$.

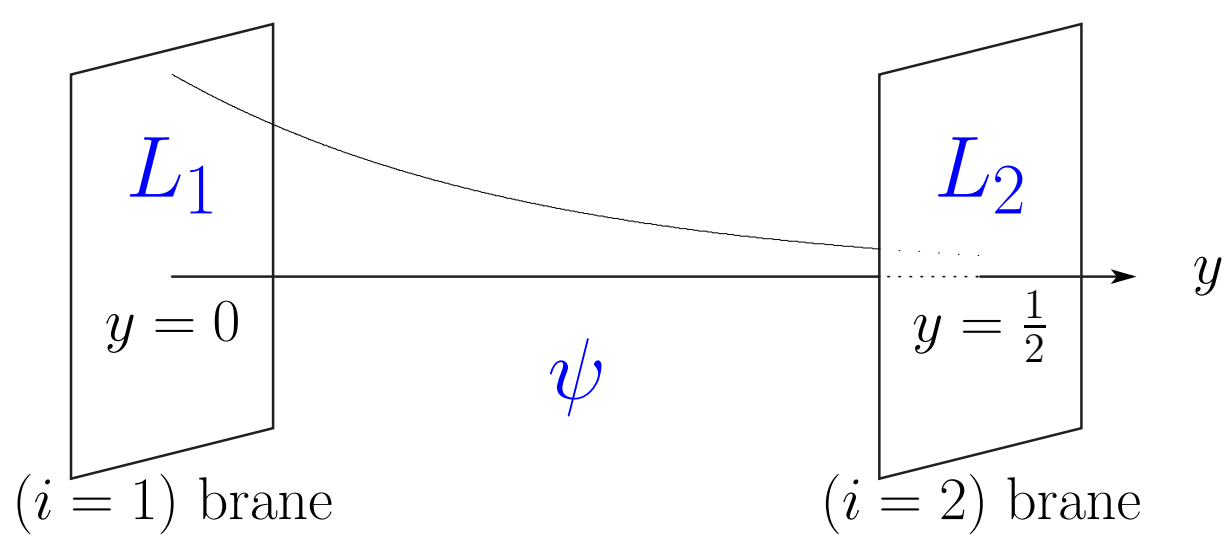

FIG. 2. Four-fermion interaction model in R.-S. background. 


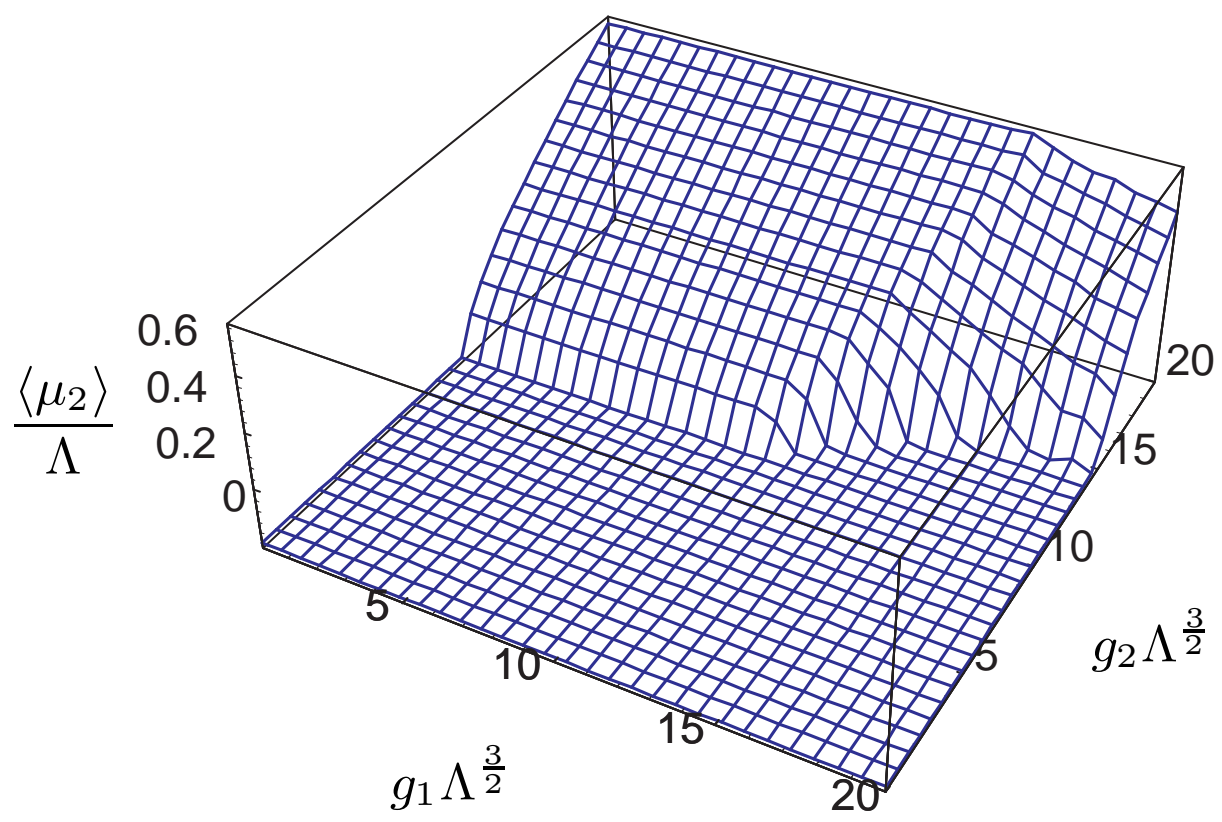

FIG. 3. $\left\langle\mu_{2}\right\rangle$ as a function of $g_{1}, g_{2}$.

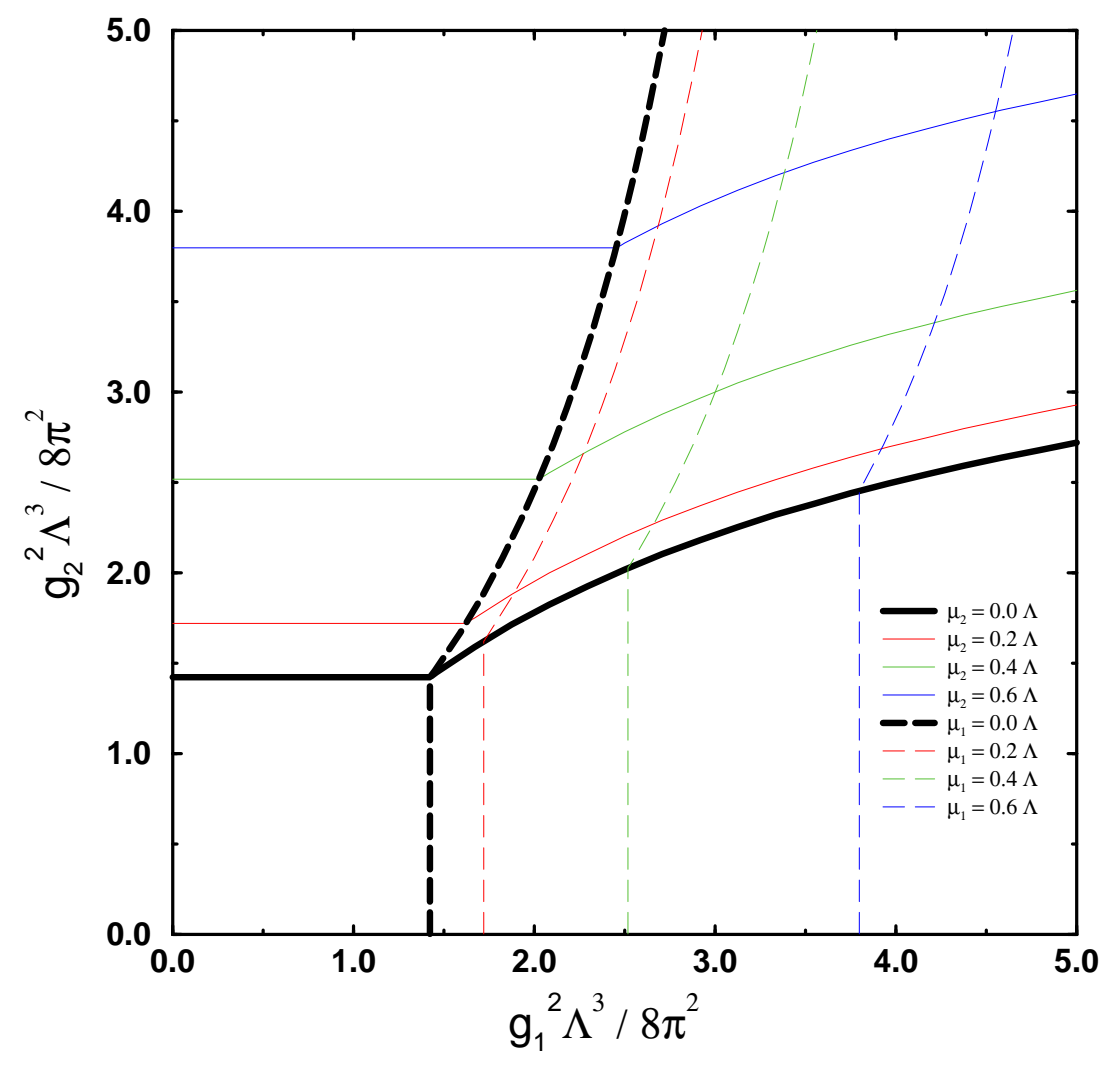

FIG. 4. The phase structure of the vacuum. 